\title{
Distributed Back-Stepping Consensus Protocol for Attitude Synchronization and Tracking on Undirected Graphs
}

DOI:

10.1142/S230138501940003X

\section{Document Version}

Accepted author manuscript

Link to publication record in Manchester Research Explorer

Citation for published version (APA):

Yu, Y., Li, Z., Yang, Z., \& Ding, Z. (2019). Distributed Back-Stepping Consensus Protocol for Attitude Synchronization and Tracking on Undirected Graphs. Unmanned Systems, 07(01), 25-32.

https://doi.org/10.1142/S230138501940003X

\section{Published in:}

Unmanned Systems

\section{Citing this paper}

Please note that where the full-text provided on Manchester Research Explorer is the Author Accepted Manuscript or Proof version this may differ from the final Published version. If citing, it is advised that you check and use the publisher's definitive version.

\section{General rights}

Copyright and moral rights for the publications made accessible in the Research Explorer are retained by the authors and/or other copyright owners and it is a condition of accessing publications that users recognise and abide by the legal requirements associated with these rights.

\section{Takedown policy}

If you believe that this document breaches copyright please refer to the University of Manchester's Takedown Procedures [http://man.ac.uk/04Y6Bo] or contact uml.scholarlycommunications@manchester.ac.uk providing relevant details, so we can investigate your claim.

\section{OPEN ACCESS}




\title{
To appear in Unmanned Systems
}

Unmanned Systems, Vol. 0, No. 0 (2018) 1-8

(C) World Scientific Publishing Company

\section{Distributed Back-stepping Consensus Protocol for Attitude Synchronization and Tracking on undirected Graphs}

\author{
Yuanjin $\mathrm{Yu}^{a}$, Zhenhong $\mathrm{Li}^{b}$, Zhaohua Yang ${ }^{c}$, Zhengtao Ding ${ }^{b, *}$ \\ ${ }^{a}$ School of Mechanical Engineering, University of Science and Technology Beijing, Beijing 100083, P. R. China \\ ${ }^{b}$ School of Electrical and Electronic Engineering, University of Manchester, Sackville Street Building, Manchester M13 \\ 9PL, UK \\ E-mail: zhengtao.ding@manchester.ac.uk \\ ${ }^{c}$ School of Instrumentation Science and Opto-electronics Engineering, Beihang University, Beijing 100191, P. R. China
}

\begin{abstract}
This paper investigates the attitude synchronization and tracking problem for multiple spacecraft with an arbitrary differentiable reference. Considering the reference as a leader, the attitude synchronization and tracking problem is then transformed into a leaderfollowing problem. Based on the quaternion representation of the attitude, the attitude tracking error dynamics and kinematics are derived. Treating the dynamics and kinematics as two cascaded subsystems, a back-stepping consensus protocol is designed, and its stability is analysed using Lyapunov theorem. The proposed protocol is fully distributed since only the local and neighbouring information are used. Finally, numerical simulations are performed to verify the effectiveness of the proposed protocol.
\end{abstract}

Keywords: Multi-agent systems; Attitude synchronization; Attitude tracking; Consensus.

\section{Introduction}

Attitude synchronization is to precisely maintain the relative attitude between spacecraft and is critical in many spacecraft formation missions. ${ }^{1}$ Due to the potential applications for space-based observer, attitude synchronization of multiple spacecraft, a specified formation control of multiagent systems, has attracted much attention. In general, the existing approaches may be classified into three types: ${ }^{2}$ leader-following, ${ }^{3}$ behavioral, ${ }^{4}$ and virtual structure. ${ }^{5}$

A fully distributed method only uses the local and neighbouring information, which is more challenging than the centralized method. There are many representations of spacecraft attitude such as Euler angle, transformation matrices, and quaternions. ${ }^{6}$ The attitude feedback control that used quaternion only was designed for a leader-following attitude consensus problem. ${ }^{7}$ The distributed cooperative method in ${ }^{8}$ used modified rodriguez parameters only for controller design and emerged the time-varying reference attitude to a virtual leader. Instead of attitude measurements, line-of-sight measurements was used in controller design. ${ }^{9}$ This method is not a distributed method and the constraints of line-of-sight measurements were not taken into account. Consensus control is an effective method in formation control by applying graph theory, ${ }^{10-14}$ and was extended to distributed disturbance observer. ${ }^{15}$ Besides general stable formation control problem, the finite-time convergence $^{16,17}$ and time delay ${ }^{18}$ were further studied.
This work concentrates on the attitude synchronization and tracking problem with arbitrary differentiable references. In leader-following formation control, at least one agent was set as the leader and the other agents were the followers aiming to maintain a relative state to the leader. ${ }^{3}$ The attitude synchronization and tracking problem is transformed into a leader-following problem while the reference attitude is regarded as the leader. Because of the cascaded structure of dynamics and kinematics of a spacecraft, back-stepping control method was applied in attitude control. ${ }^{19,20}$ To realize attitude synchronization, the sliding-mode vector simultaneously includes the attitude error with respect to the reference and the relative errors between spacecraft was designed in. ${ }^{1}$ Inspired by these methods, a fully distributed back-stepping consensus protocol is designed to achieve attitude synchronization and tracking under an undirected communication. After treating the reference attitude as the virtual leader, the attitude error with respect to the reference becomes a relative error. Then the control objective is to regulate the relative errors only. A back-stepping method is designed using the relative attitude errors, make the controller be fully distributed and no need of the neighbour's attitude velocity. Numerical simulations in two different topologies are constructed to demonstrate this method. The results indicate that this method can realize attitude synchronization and tracking.

The remainder contents are organized as follows: In 
Section 2, some preliminaries were presented and the problem statement was introduced. In Section 3, the distributed back-stepping consensus protocol was designed in detail. Then, many simulations were performed to demonstrate the designed method in Section 4. Finally, the conclusions were drawn in Section 5.

\section{Preliminaries and Problem Formulation}

Attitude synchronization and tracking problem is considered in this work. In this section, some preliminaries are presented and then the problem formulation is drawn.

\subsection{Quaternion}

There are many representations of attitude, of which Euler angles, quaternions, and transformation matrices are widely used. To avoid the singularity, quaternion is chosen in

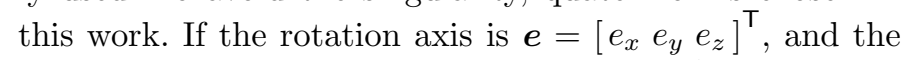
rotation angle is $\Phi$, quaternion is defined as, ${ }^{6}$

$$
\boldsymbol{q}=\left[\begin{array}{l}
q_{0} \\
q_{1} \\
q_{2} \\
q_{3}
\end{array}\right]=\left[\begin{array}{c}
\cos \left(\frac{\Phi}{2}\right) \\
e_{x} \sin \left(\frac{\Phi}{2}\right) \\
e_{y} \sin \left(\frac{\Phi}{2}\right) \\
e_{z} \sin \left(\frac{\Phi}{2}\right)
\end{array}\right]
$$

Quaternion is a four elements vector. The four elements satisfy $q_{0}^{2}+q_{1}^{2}+q_{2}^{2}+q_{3}^{2}=1$ and are divided into a scaler $q_{0}$ and a vector $\boldsymbol{q}_{v}=\left[\begin{array}{lll}q_{1} & q_{2} & q_{3}\end{array}\right]^{\top}$.

The transformation from quaternion to transformation matrix, also referred as direction cosine matrix, is expressed as,

$$
\begin{aligned}
& \mathbf{R}(\boldsymbol{q}) \\
& =\left[\begin{array}{ccc}
q_{0}^{2}-q_{1}^{2}-q_{2}^{2}-q_{3}^{2} & 2\left(q_{0} q_{3}+q_{1} q_{2}\right) & 2\left(q_{1} q_{3}-q_{0} q_{2}\right) \\
2\left(q_{1} q_{2}-q_{0} q_{3}\right) & q_{0}^{2}-q_{1}^{2}+q_{2}^{2}-q_{3}^{2} & 2\left(q_{0} q_{1}+q_{2} q_{3}\right) \\
2\left(q_{0} q_{2}+q_{1} q_{3}\right) & 2\left(q_{2} q_{3}-q_{0} q_{1}\right) & q_{0}^{2}-q_{1}^{2}-q_{2}^{2}+q_{3}^{2}
\end{array}\right] \\
& =\left(q_{0}^{2}-\boldsymbol{q}_{v}^{\top} \boldsymbol{q}_{v}\right) \mathbf{I}_{3 \times 3}+2 \boldsymbol{q}_{v} \boldsymbol{q}_{v}^{\top}-2 q_{0} \boldsymbol{q}_{v} \times
\end{aligned}
$$

where $\mathbf{I}_{3 \times 3}$ is a 3-order identity matrix, $\mathbf{R}(\boldsymbol{q})$ is the transformation matrix and $\boldsymbol{q}_{v}^{\times}$is a skew-symmetric matrix defined as

$$
\boldsymbol{q}_{v} \times=\left[\begin{array}{ccc}
0 & -q_{3} & q_{2} \\
q_{3} & 0 & -q_{1} \\
-q_{2} & q_{1} & 0
\end{array}\right]
$$

The transformation from transformation matrix to a 3-1-2 Euler angle is expressed as

$$
\begin{aligned}
& \varphi=\arcsin \left(R(q)_{23}\right) \\
& \theta=\arctan \left(-\frac{R(q)_{13}}{R(q)_{33}}\right) \\
& \psi=\arctan \left(-\frac{R(q)_{21}}{R(q)_{22}}\right)
\end{aligned}
$$

where $\psi, \varphi$, and $\theta$ are the Euler angles that rotates in order of $z-, x-$, and $y$-axis, which are referred as yaw, roll, and pitch angle, respectively. $R(q)_{i j}$ denotes the $i$-th row and $j$-th column element of matrix $\mathbf{R}(\boldsymbol{q})$.

\subsection{Rigid body attitude dynamics and kinematics}

Ignoring the flexible parts, assume all the spacecraft here are rigid body. The basic concepts of attitude dynamics are introduced. The attitude dynamics and kinematics of a rigid body can be expressed as, ${ }^{6}$

$$
\begin{aligned}
& \mathbf{J} \dot{\boldsymbol{\omega}}=-\boldsymbol{\omega}^{\times} \mathbf{J} \boldsymbol{\omega}+\boldsymbol{T}_{c}+\boldsymbol{T}_{d} \\
& \dot{q}_{0}=-\frac{1}{2} \boldsymbol{q}_{v}^{\top} \boldsymbol{\omega} \\
& \dot{\boldsymbol{q}}_{v}=\frac{1}{2}\left(\boldsymbol{q}_{v}^{\times}+q_{0} \mathbf{I}_{3 \times 3}\right) \boldsymbol{\omega}
\end{aligned}
$$

where $\boldsymbol{q}=\left[\begin{array}{llll}q_{0} & q_{1} & q_{2} & q_{3}\end{array}\right]^{\top}$ denotes the attitude quaternion, $q_{0} \in \mathbb{R}$ and $\boldsymbol{q}_{v}=\left[\begin{array}{lll}q_{1} & q_{2} & q_{3}\end{array}\right]^{\top} \in \mathbb{R}^{3}$ denote the scalar part and the vector part of quaternion, respectively. $\mathbf{J} \in \mathbb{R}^{3 \times 3}$ denotes the inertia matrix. $\boldsymbol{\omega} \in \mathbb{R}^{3}$ denotes the attitude angular velocity represented in body-fixed frame and with respect to the inertial frame. Note that $\boldsymbol{V}^{\times} \boldsymbol{Y}=\boldsymbol{V} \times \boldsymbol{Y}$ is the cross-product of $\boldsymbol{V}, \boldsymbol{Y} \in \mathbb{R}^{3} . \boldsymbol{T}_{c} \in \mathbb{R}^{3}$ and $\boldsymbol{T}_{d} \in \mathbb{R}^{3}$ denote the control torque and disturbance torque, respectively.

\subsection{Rigid body attitude-tracking error dynamics and kinetics}

The attitude of all the spacecraft are expected to approach a same reference attitude. Let $\boldsymbol{q}_{d}=\left[q_{0, d} \boldsymbol{q}_{v, d}^{\top}\right]^{\top}$ be the reference quaternion, $\boldsymbol{\omega}_{d} \in \mathbb{R}^{3}$ be the reference angular velocity represented in a reference frame and with respect to the inertial frame, the quaternion error $\boldsymbol{q}_{e}$ and velocity error $\tilde{\boldsymbol{\omega}}_{e}$ are expressed as

$$
\boldsymbol{q}_{e}=\boldsymbol{q}_{d}{ }^{-1} \boldsymbol{q}=\left[\begin{array}{c}
\boldsymbol{q}^{\top} \boldsymbol{q}_{d} \\
q_{0, d} \boldsymbol{q}_{v}-q_{0} \boldsymbol{q}_{v, d}+\left(\boldsymbol{q}_{v}\right)^{\times} \boldsymbol{q}_{v, d}
\end{array}\right]
$$

where $q_{0, e} \in \mathbb{R}$ and $\boldsymbol{q}_{v, e}=\left[q_{1, e} q_{2, e} q_{3, e}\right]^{\top} \in \mathbb{R}^{3}$ denote the scalar part and the vector part of error quaternion, respectively, $\mathbf{R}\left(\boldsymbol{q}_{e}\right)$ is the orientation cosine matrix from the reference frame to the body-fixed frame, which is expressed as $\mathbf{R}\left(\boldsymbol{q}_{e}\right)=\left(q_{0, e}^{2}-\boldsymbol{q}_{v, e^{\top}} \boldsymbol{q}_{v, e}\right) \mathbf{I}_{3 \times 3}+2 \boldsymbol{q}_{v, e} \boldsymbol{q}_{v, e}{ }^{\mathrm{\top}}-2 q_{0, e} \boldsymbol{q}_{v, e} \times$. The error dynamics and kinetics can be expressed as, $\mathbf{J} \dot{\boldsymbol{\omega}}_{e}=-\boldsymbol{\omega}^{\times} \mathbf{J} \boldsymbol{\omega}+\mathbf{J} \boldsymbol{\omega}_{e}^{\times} \mathbf{R}\left(q_{e}\right) \boldsymbol{\omega}_{d}-\mathbf{J R}\left(q_{e}\right) \dot{\boldsymbol{\omega}}_{d}+\boldsymbol{T}_{c}+\boldsymbol{T}_{d}$ $\dot{q}_{0, e}=-\frac{1}{2}\left(\boldsymbol{q}_{v, e}\right)^{\top} \boldsymbol{\omega}_{e}$ $\dot{\boldsymbol{q}}_{v, e}=\frac{1}{2}\left(\boldsymbol{q}_{v, e} \times q_{0, e} \mathbf{I}_{3 \times 3}\right) \boldsymbol{\omega}_{e}$

\subsection{Graph theory}

The communications between agents can be described by a graph $\mathcal{G}(\mathcal{V}, \mathcal{E})$, where $\mathcal{V}$ and $\mathcal{E}$ denote the agents and their communication connections, respectively. For the undirected graph, $(i, j) \in \mathcal{E}$ represents the $i$-th agent can exchange informations with the $j$-th agent. For a group of $N$ agents, 
the associated adjacency matrix is defined as $\mathcal{A}=\left[a_{i j}\right]$, where $a_{i j}>0$ if $(i, j) \in \mathcal{E}$. In undirected graphs, we have $a_{i j}=a_{j i}$. An undirected graph is connected if there exists a path between any two agents.

\subsection{Problem formulation}

In this work, we consider a group of $N$ spacecraft that are rigid. According to the rigid body dynamics in (4), the attitude dynamics and kinematics of the $i$-th rigid spacecraft can be expressed as

$$
\begin{aligned}
& \mathbf{J}_{i} \dot{\boldsymbol{\omega}}_{i}=-\boldsymbol{\omega}_{i}^{\times} \mathbf{J}_{i} \boldsymbol{\omega}_{i}+\boldsymbol{T}_{c i}+\boldsymbol{T}_{d i} \\
& \dot{q}_{0, i}=-\frac{1}{2} \boldsymbol{q}_{v, i}^{\top} \boldsymbol{\omega}_{i} \\
& \dot{\boldsymbol{q}}_{v, i}=\frac{1}{2}\left(\boldsymbol{q}_{v, i}^{\times}+q_{0, i} \mathbf{I}_{3 \times 3}\right) \boldsymbol{\omega}_{i} \quad(i=1,2, \cdots, N)
\end{aligned}
$$

Consider a situation that the spacecraft group are required to approach a common expected attitude. The objective is to achieve attitude synchronization and tracking for multiple spacecraft under the following three assumptions.

Assumption 1. The reference attitude is arbitrary differentiable.

The situation that the dynamics of the tracking target are unknown is adapt to many practical attitude formation control.

Assumption 2. The information exchanging among the spacecraft is specified by a connected undirected graph.

In this work, the relative attitude errors are only used in controller design. Thus the exchanged information is the spacecraft attitude that is obtained by the attitude sensors such as gyroscopes, sun sensor, and star sensor.

Assumption 3. At least one spacecraft has the information of reference attitude.

\section{Controller Design}

The objective is to achieve attitude tracking. The attitude of all the spacecraft are also expected to synchronize with their neighbours. Then, the attitude synchronization is treated as attitude tracking for each spacecraft, thus the relative attitude between a spacecraft and one of its neighbour is treated as the attitude error. Define $\boldsymbol{q}_{i j}$ and $\boldsymbol{\omega}_{i j}$ be the attitude and velocity error between the $i$-th spacecraft and the attitude of the $j$-th spacecraft, respectively, where $i, j=1,2, \cdots, N$. Using the definition of tracking error (5), we have

$$
\begin{aligned}
& \boldsymbol{q}_{i j}=\boldsymbol{q}_{j}{ }^{-1} \boldsymbol{q}_{i}=\left[\begin{array}{c}
\boldsymbol{q}_{i}^{\top} \boldsymbol{q}_{j} \\
q_{0, j} \boldsymbol{q}_{v, i}-q_{0, i} \boldsymbol{q}_{v, j}+\left(\boldsymbol{q}_{v, i}\right)^{\times} \boldsymbol{q}_{v, j}
\end{array}\right] \\
& \boldsymbol{\omega}_{i j}=\boldsymbol{\omega}_{i}-\mathbf{R}\left(\boldsymbol{q}_{i j}\right) \boldsymbol{\omega}_{j}
\end{aligned}
$$

where $\mathbf{R}\left(\boldsymbol{q}_{i j}\right)$ is the attitude transformation matrix from the coordinate of the $j$-th spacecraft to that of the $i$-th spacecraft, which is expressed as $\mathbf{R}\left(\boldsymbol{q}_{i j}\right)=$ $\left(q_{0, i j}^{2}-\boldsymbol{q}_{v, i j}{ }^{\top} \boldsymbol{q}_{v, i j}\right) \mathbf{I}_{3 \times 3}+2 \boldsymbol{q}_{v, i j} \boldsymbol{q}_{v, i j}{ }^{\top}-2 q_{0, i j} \boldsymbol{q}_{v, i j} \times$
According to attitude-tracking error dynamics in (6), the error dynamics can be expressed as

$$
\begin{aligned}
\mathbf{J}_{i} \dot{\boldsymbol{\omega}}_{i j}= & -\boldsymbol{\omega}_{i}^{\times} \mathbf{J}_{i} \boldsymbol{\omega}_{i}+\mathbf{J}_{i} \boldsymbol{\omega}_{i j} \times \mathbf{R}\left(\boldsymbol{q}_{i j}\right) \boldsymbol{\omega}_{j}-\mathbf{J}_{i} \mathbf{R}\left(\boldsymbol{q}_{i j}\right) \dot{\boldsymbol{\omega}}_{j} \\
& +\boldsymbol{T}_{c}+\boldsymbol{T}_{d} \\
\dot{q}_{0, i j}= & -\frac{1}{2}\left(\boldsymbol{q}_{v, j i}\right)^{\top} \boldsymbol{\omega}_{i j} \\
\dot{\boldsymbol{q}}_{v, i j}= & \frac{1}{2}\left(\boldsymbol{q}_{v, i j}{ }^{\times}+q_{0, i j} \mathbf{I}_{3 \times 3}\right) \boldsymbol{\omega}_{i j} \quad(i, j=1,2, \cdots, N)
\end{aligned}
$$

In this work, the reference quaternion is treated as a leader spacecraft indexed by 0 , which makes the attitude synchronization becomes a leader-follower formation problem. The leader is specified as a special spacecraft with $\boldsymbol{q}_{0}=\boldsymbol{q}_{d}$ and $\boldsymbol{\omega}_{0}=\mathbf{0}$. Thus when $i=0$ or $j=0$, (8) is also satisfied. Under Assumptions 2 and 3, the new group of $N+1$ spacecraft also connected by a connected undirected graph.

For the $i$-th spacecraft, attitude quaternion $\boldsymbol{q}_{i}$ is the output variable and $a_{i j} \boldsymbol{q}_{i j}$ is available in distributed controller design on the basis of connected graph. A distributed control protocol is designed as

$$
\begin{aligned}
\boldsymbol{T}_{c i}= & -\eta_{i} \tilde{\boldsymbol{\omega}}_{i}-\left(\sum_{j=0}^{N} a_{i j}\left(\boldsymbol{q}_{v, i j}\right)\right)+\boldsymbol{\omega}_{i}^{\times} \mathbf{J}_{i} \boldsymbol{\omega}_{i}-d_{i} \operatorname{sign}\left(\tilde{\boldsymbol{\omega}}_{i}\right) \\
& -\mathbf{J}_{i} \operatorname{diag}\left(\frac{\alpha_{i} \beta_{i}}{1+\left(\beta_{i} \sum_{j=0}^{N} a_{i j} \boldsymbol{q}_{v, i j}\right)^{2}}\right) \sum_{j=0}^{N} a_{i j} \dot{\boldsymbol{q}}_{v, i j} \\
& (i=1,2, \cdots, N)
\end{aligned}
$$

where $\tilde{\boldsymbol{\omega}}_{i}=\boldsymbol{\omega}_{i}-\boldsymbol{\omega}_{i}{ }^{d}, \boldsymbol{\omega}_{i}{ }^{d}=-\alpha_{i} \arctan \left(\beta_{i} \sum_{j=0}^{N} a_{i j} \boldsymbol{q}_{v, i j}\right), \alpha_{i}$, $\beta_{i}$, and $\eta_{i}$ are positive constant, $d_{i}$ is positive constant and satisfy $d_{i}>\left\{\left\|\boldsymbol{T}_{d i}\right\|\right\}_{\max }$.

Theorem1: Consider a group of attitude system (7). Suppose Assumptions 1-3 hold. Consensus tracking is achieved with the controller in (10).

Proof. The Lyapunov function candidate is initially chosen as,

$$
V_{1}=\sum_{i=0}^{N} \sum_{j=0}^{N} a_{i j}\left(1-q_{0, i j}\right)
$$

Derivative of (11) is

$$
\begin{aligned}
\dot{V}_{1} & =\frac{1}{2} \sum_{i=0}^{N} \sum_{j=0}^{N} a_{i j}\left(\boldsymbol{q}_{v, i j}\right)^{\top} \boldsymbol{\omega}_{i j} \\
& =\frac{1}{2} \sum_{i=0}^{N} \sum_{j=0}^{N} a_{i j}\left(\boldsymbol{q}_{v, i j}\right)^{\top} \boldsymbol{\omega}_{i}-a_{i j}\left(\boldsymbol{q}_{v, i j}\right)^{\top} \boldsymbol{\omega}_{j} \\
& =\frac{1}{2} \sum_{i=0}^{N} \sum_{j=0}^{N} a_{i j}\left(\boldsymbol{q}_{v, i j}\right)^{\top} \boldsymbol{\omega}_{i}-a_{j i}\left(\boldsymbol{q}_{v, j i}\right)^{\top} \boldsymbol{\omega}_{i} \\
& =\sum_{i=0}^{N} \sum_{j=0}^{N} a_{i j}\left(\boldsymbol{q}_{v, i j}\right)^{\top} \boldsymbol{\omega}_{i}
\end{aligned}
$$


The virtual input is designed as,

$$
\begin{gathered}
\boldsymbol{\omega}_{i}{ }^{d}=-\alpha_{i} \arctan \left(\beta_{i} \sum_{j=0}^{N} a_{i j} \boldsymbol{q}_{v, i j}\right) \\
(i=1,2, \cdots, N)
\end{gathered}
$$

where $\alpha_{i}$ and $\beta_{i}$ are positive constants. Substituting (13) into (12), we have

$$
\dot{V}_{1}=\frac{1}{2} \sum_{i=1}^{N}\left(\sum_{j=0}^{N} a_{i j}\left(\boldsymbol{q}_{v, i j}\right)^{\top}\right) \boldsymbol{\omega}_{i}{ }^{d} \leq 0
$$

In attitude dynamics, $\boldsymbol{\omega}_{i}$ is treated as the output, $\tilde{\boldsymbol{\omega}}_{i}=\boldsymbol{\omega}_{i}-\boldsymbol{\omega}_{i}{ }^{d}$ denotes the tracking error, we have

$$
\begin{aligned}
\mathbf{J}_{i} \dot{\tilde{\boldsymbol{\omega}}}_{i}= & \mathbf{J}_{i} \dot{\boldsymbol{\omega}}_{i}-\mathbf{J}_{i} \dot{\boldsymbol{\omega}}_{i}^{d} \\
= & -\boldsymbol{\omega}_{i}^{\times} \mathbf{J}_{i} \boldsymbol{\omega}_{i}+\boldsymbol{T}_{c i}+\boldsymbol{T}_{d i} \\
& +\mathbf{J}_{i} \operatorname{diag}\left(\frac{\alpha_{i} \beta_{i}}{1+\left(\beta_{i} \sum_{j=0}^{N} a_{i j} \boldsymbol{q}_{v, i j}\right)^{2}}\right) \sum_{j=0}^{N} a_{i j} \dot{\boldsymbol{q}}_{v, i j}
\end{aligned}
$$

The Lyapunov function candidate is then chosen as,

$$
V_{2}=\sum_{i=0}^{N} \sum_{j=0}^{N} a_{i j}\left(1-q_{0, i j}\right)+\sum_{i=1}^{N} \frac{1}{2} \tilde{\boldsymbol{\omega}}_{i}^{\top} \mathbf{J}_{i} \tilde{\boldsymbol{\omega}}_{i}
$$

Derivative of (16) is

$$
\begin{aligned}
\dot{V}_{2}= & \frac{1}{2} \sum_{i=1}^{N}\left(\sum_{j=0}^{N} a_{i j}\left(\boldsymbol{q}_{v, i j}\right)^{\top}\right) \boldsymbol{\omega}_{i}{ }^{d} \\
& +\frac{1}{2} \sum_{i=1}^{N}\left(\sum_{j=0}^{N} a_{i j}\left(\boldsymbol{q}_{v, i j}\right)^{\top}\right) \tilde{\boldsymbol{\omega}}_{i}+\frac{1}{2} \sum_{i=1}^{N} \tilde{\boldsymbol{\omega}}_{i}^{\top} \mathbf{J}_{i} \dot{\tilde{\boldsymbol{\omega}}}_{i} \\
= & \frac{1}{2} \sum_{i=1}^{N}\left(\sum_{j=0}^{N} a_{i j}\left(\boldsymbol{q}_{v, i j}\right)^{\top}\right) \boldsymbol{\omega}_{i}{ }^{d} \\
& +\frac{1}{2} \sum_{i=1}^{N} \tilde{\boldsymbol{\omega}}_{i}^{\top}\left[\left(\sum_{j=0}^{N} a_{i j}\left(\boldsymbol{q}_{v, i j}\right)\right)-\boldsymbol{\omega}_{i}^{\times} \mathbf{J}_{i} \boldsymbol{\omega}_{i}+\boldsymbol{T}_{c i}+\boldsymbol{T}_{d i}\right. \\
& \left.+\mathbf{J}_{i} \operatorname{diag}\left(\frac{\alpha_{i} \beta_{i}}{1+\left(\beta_{i} \sum_{j=0}^{N} a_{i j} \boldsymbol{q}_{v, i j}\right)^{2}}\right) \sum_{j=0}^{N} a_{i j} \dot{\boldsymbol{q}}_{v, i j}\right]
\end{aligned}
$$

Substituting (10) into (17), we have

$$
\dot{V}_{2}=\dot{V}_{1}+\frac{1}{2} \sum_{i=0}^{N} \tilde{\boldsymbol{\omega}}_{i}^{\top}\left[-\eta_{i} \tilde{\boldsymbol{\omega}}_{i}-d_{i} \operatorname{sign}\left(\tilde{\boldsymbol{\omega}}_{i}\right)+\boldsymbol{T}_{d i}\right] \leq 0
$$

With controller designed in (10), $V_{2}$ will approaches 0 , means $\lim _{t \rightarrow \infty} a_{i j}\left(1-q_{0, i j}\right)=0$ for $i, j=1,2, \cdots, N$. The relative attitude between any two neighboured spacecraft converges, then the attitude synchronization is achieved.

Remark. The protocol in (10) is fully distributed. Each spacecraft only uses itself information and the attitude of their neighbours.

\section{Simulations and discussion}

Simulations are performed to validate the effectiveness of the proposed protocol. Consider a group of 4 spacecraft whose attitude dynamics is in the form as (7), with $\mathbf{J}_{i}=\left[\begin{array}{ccc}100 & 0 & 0 \\ 0 & 100 & 0 \\ 0 & 0 & 200\end{array}\right] \mathrm{kg} \cdot \mathrm{m}^{2}$. Simulations are performed in two different communication topologies, shown in Figure 1.

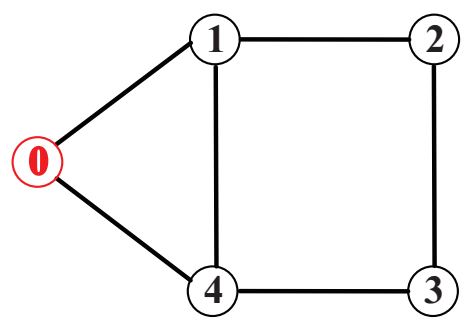

(a)Topology 1

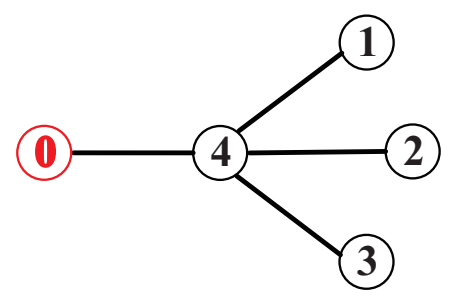

(b)Topology 2

Fig. 1. Connection topology

In Figure 1, the circle indexed by 0 denotes the leader and the other four circles are followers. In Figure 1(a), the four followers are connected in circle. By contrast in Figure 1(b), the four followers are connected in star. The adjacency matrices of the two topologies are given respectively as

$$
\mathcal{A}_{1}=\left[\begin{array}{lllll}
0 & 1 & 0 & 0 & 1 \\
1 & 0 & 1 & 0 & 1 \\
0 & 1 & 0 & 1 & 0 \\
0 & 0 & 1 & 0 & 1 \\
1 & 1 & 0 & 1 & 0
\end{array}\right], \mathcal{A}_{2}=\left[\begin{array}{lllll}
0 & 0 & 0 & 0 & 1 \\
0 & 0 & 1 & 1 & 1 \\
0 & 1 & 0 & 0 & 0 \\
0 & 1 & 0 & 0 & 0 \\
1 & 1 & 0 & 0 & 0
\end{array}\right]
$$

The reference attitude, referred as that of the leader, is set in two cases, (1) Constant case: all three Euler angles are constants; (2) Harmonic case: all three Euler angles are sinusoidal.

The initial attitude velocity are set as $\boldsymbol{\omega}_{1}(0)=\boldsymbol{\omega}_{2}(0)=$ $\boldsymbol{\omega}_{3}(0)=\boldsymbol{\omega}_{4}(0)=[0,0,0]^{\top} \mathrm{rad} / \mathrm{s}$, and the initial quaternion of the four spacecraft are randomly chosen as,

$$
\boldsymbol{q}_{1}(0)=\left[\begin{array}{l}
0.9912 \\
0.1018 \\
0.0730 \\
0.0419
\end{array}\right], \boldsymbol{q}_{2}(0)=\left[\begin{array}{l}
0.9978 \\
0.0156 \\
0.0358 \\
0.0529
\end{array}\right]
$$




$$
\boldsymbol{q}_{3}(0)=\left[\begin{array}{c}
0.9979 \\
-0.0529 \\
-0.0339 \\
-0.0156
\end{array}\right], \boldsymbol{q}_{4}(0)=\left[\begin{array}{c}
0.9917 \\
-0.0419 \\
-0.0657 \\
-0.1018
\end{array}\right]
$$

The parameters of the controller are set as: $\eta_{1}=$ $\eta_{2}=\eta_{3}=\eta_{4}=100, d_{1}=d_{2}=d_{3}=d_{4}=0.1$, $\alpha_{1}=\alpha_{2}=\alpha_{3}=\alpha_{4}=2$, and $\beta_{1}=\beta_{2}=\beta_{3}=\beta_{4}=500$.

The attitude results of the spacecraft connected in topology 1 are given in Figures 2 and 3, of which attitude is described by three Euler angles as roll, pitch, and yaw.

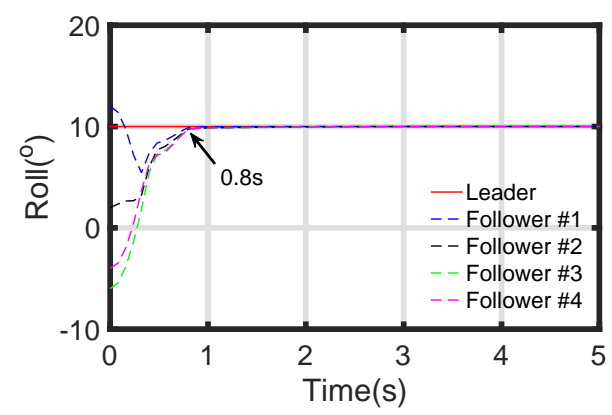

(a)Roll

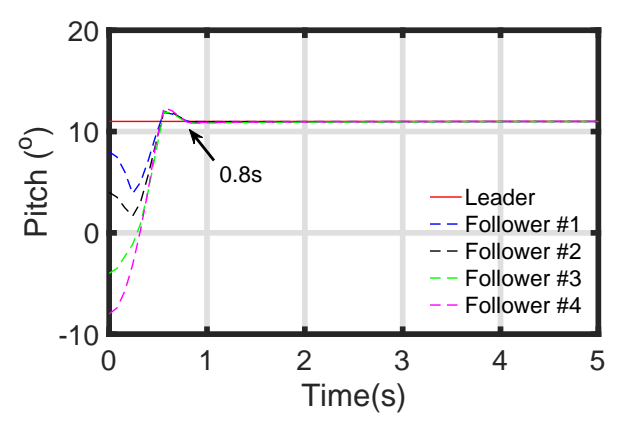

(b)Pitch

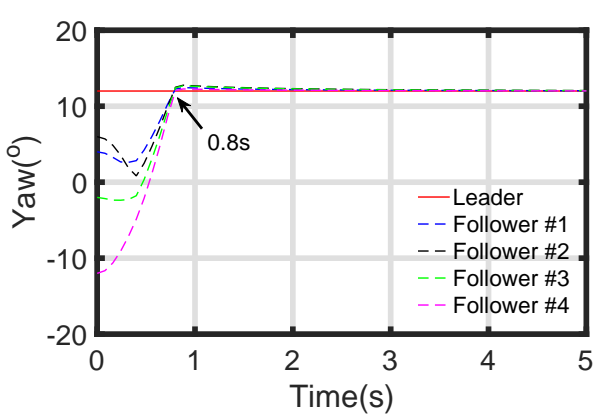

(c) Yaw

Fig. 2. Constant reference(Topology 1)
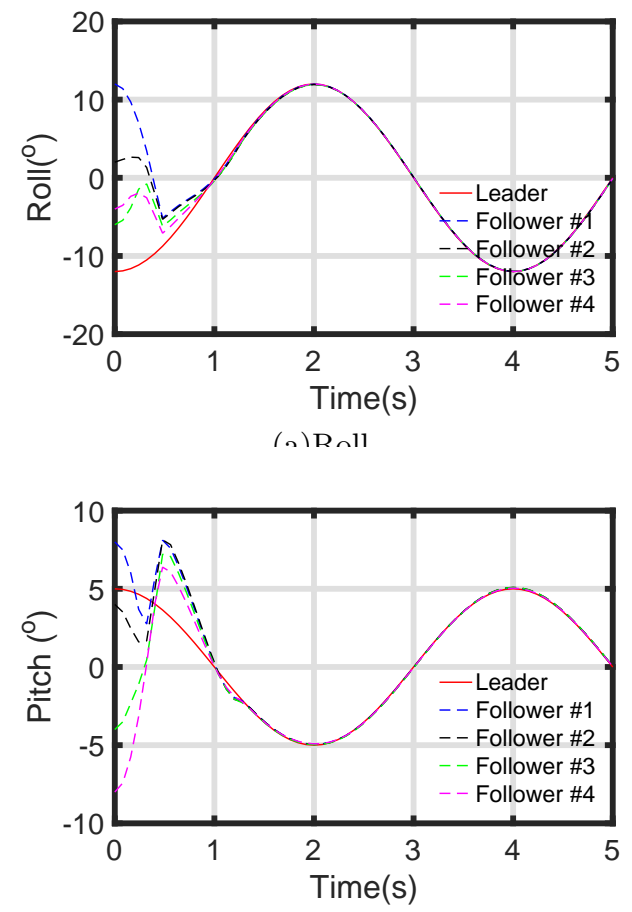

(b)Pitch

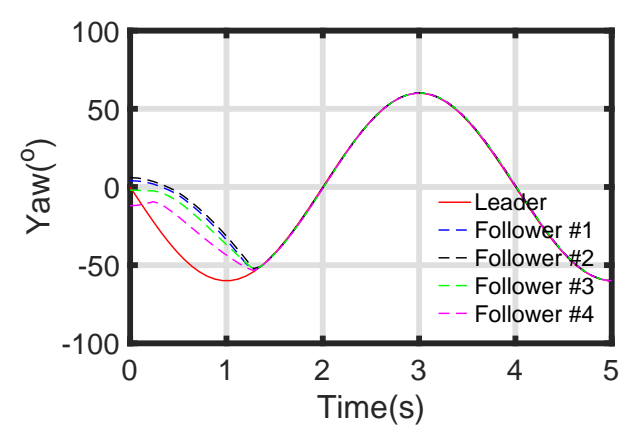

(c)Yaw

Fig. 3. Harmonic reference(Topology 1)

Figures 2 and 3 are the attitude responses when reference attitude are set as constants and harmonic, respectively. Red solid lines are reference Euler angles, the blue, black, green, and magenta dash lines are the Euler angles of the followers $1,2,3$, and 4, respectively. Figure 2 illustrates the attitude of the four spacecraft converge to the reference attitude with about $0.8 s$ convergence time. Figure 3 shows the attitude of the followers converge to the reference attitude, which achieve attitude synchronization. After that, the followers maintain attitude synchronization and their attitude stably track the reference.

The attitude results of the spacecraft connected in topology 2 are given in Figures 4 and 5 . 


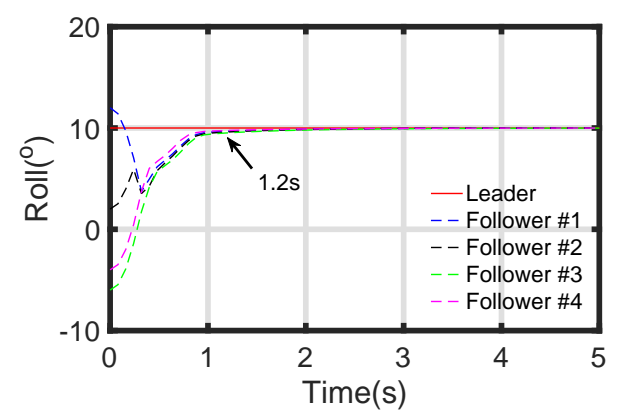

(a)Roll

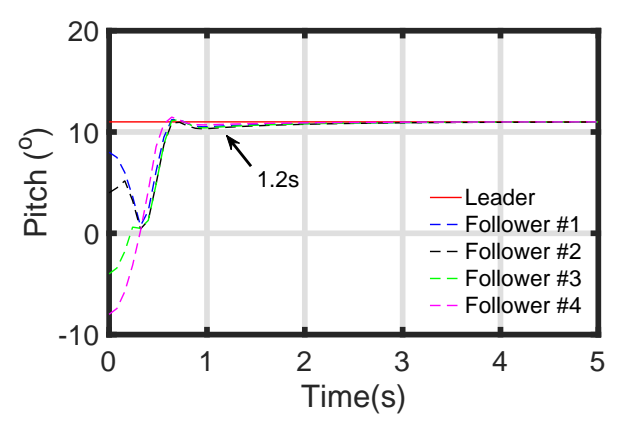

(b)Pitch

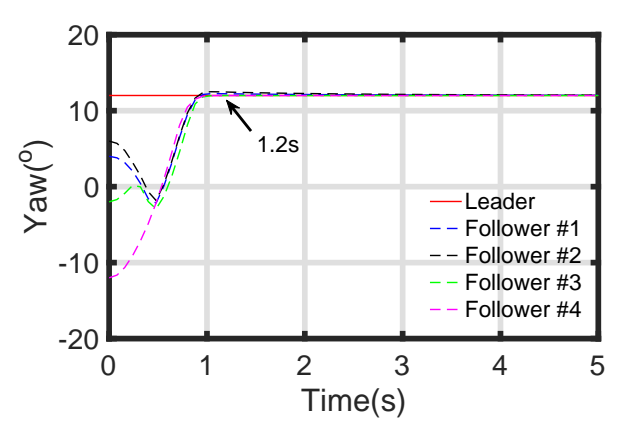

(c) Yaw

Fig. 4. Constant reference(Topology 2)

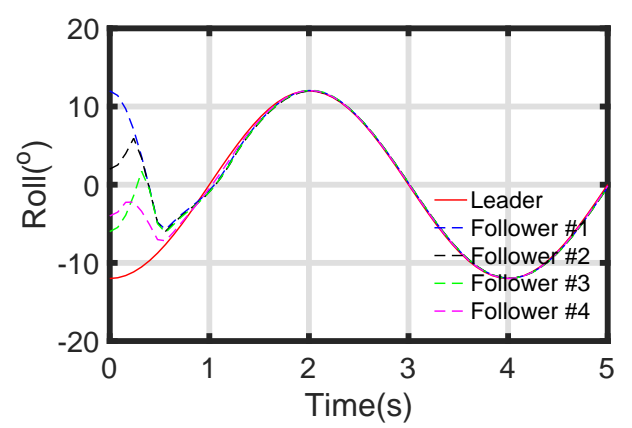

(a)Roll

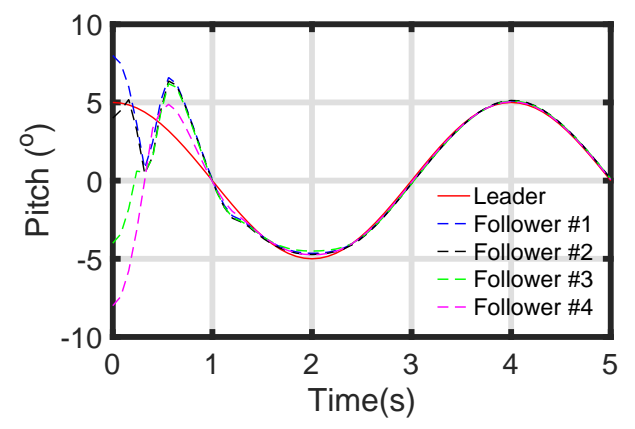

(b)Pitch

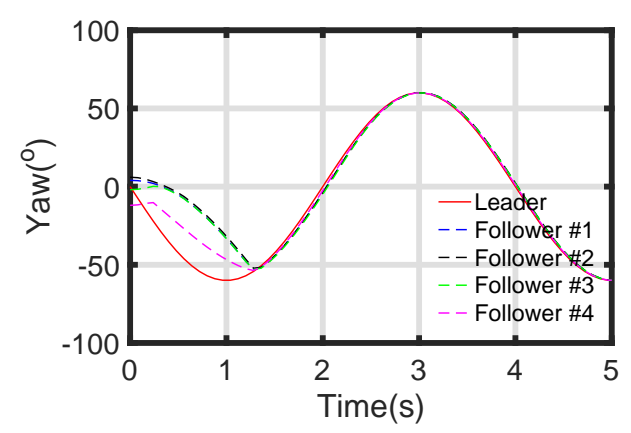

(c)Yaw

Fig. 5. Harmonic reference(Topology 2)

Figures 4 and 5 are the attitude responses when reference attitude are set as constants and harmonic, respectively. Definitions of the lines are the same with that of Figures 2 and 3. Figure 4 also shows the attitude of the followers stably track the reference attitude, but different from the results of Figure 2, dynamic responses in Figure 4 have longer convergence time, about 1.2s. Figure 5 also validates the correspond results in Figure 3 though their dynamic responses are tiny different. These results show the attitude synchronization and tracking is also achieved with a different topology.

\section{Conclusion}

This work considered an attitude synchronization and tracking problem for multiple spacecraft. It was transferred to a leader-following formation while the reference attitude was regarded as a virtual leader. Because of nonsingularity, quaternions were chosen as the representation of attitude. A back-stepping design method is applied to derive the fully distributed consensus protocol. This method does not need neighbour's attitude velocity for the controller of each spacecraft. Finally, this method was applied to synchronize tracking a time-varying reference attitude and its effectiveness was demonstrated by many numerical simulations. The proposed controller was designed on undirected graphs, a little more works should be done to extend the designed method to the directed case. 


\section{Acknowledgments}

This work was supported by the Science and Technology Facilities Council (STFC) under Grant ST/N006852/1.

\section{References}

[1] B. Wu and D. Wang, Decentralized robust adaptive control for attitude synchronization under directed communication topology, Journal of Guidance, Control, and Dynamics 34 (July-August 2011) 1276-1282.

[2] R. W. Beard, J. Lawton and F. Y. Hadaegh, A coordination architecture for spacecraft formation control, IEEE Transactions on Control Systems Technology 9 (Nov 2001) 777-790.

[3] L. Consolini, F. Morbidi, D. Prattichizzo and M. Tosques, Leadercfollower formation control of nonholonomic mobile robots with input constraints, $A u$ tomatica 44(5) (2008) 1343 - 1349.

[4] T. Balch and R. C. Arkin, Behavior-based formation control for multirobot teams, IEEE Transactions on Robotics and Automation 14 (Dec 1998) 926-939.

[5] M. A. Lewis and K.-H. Tan, High precision formation control of mobile robots using virtual structures, $A u$ tonomous Robots 4 (Oct 1997) 387-403.

[6] M. Paluszek, P. Bhatta, P. Griesemer, J. Mueller and S. Thomas, Spacecraft Attitude and Orobit Control, 2nd edn. (Princeton Satellite Systems, Inc, 1996).

[7] H. Cai and J. Huang, Leader-following attitude consensus of multiple rigid body systems by attitude feedback control, Automatica 69 (2016) 87 - 92.

[8] W. Ren, Distributed cooperative attitude synchronization and tracking for multiple rigid bodies, IEEE Transactions on Control Systems Technology 18 (March 2010) 383-392.

[9] T.-H. Wu, B. Flewelling, F. Leve and T. Lee, Spacecraft attitude-formation tracking using line-of-sight measurements, Journal of Guidance, Control, and Dynamics 40(10) (2017) 2616-2629.

[10] W. Ren, Consensus strategies for cooperative control of vehicle formations, IET Control Theory and Applications 1 (March 2007) 505-512.

[11] X. Zhang, L. Liu and G. Feng, Leadercfollower consensus of time-varying nonlinear multi-agent systems, Automatica 52 (2015) 8-14.

[12] M. Defoort, A. Polyakov, G. Demesure, M. Djemai and K. Veluvolu, Leader-follower fixed-time consensus for multi-agent systems with unknown non-linear inherent dynamics, IET Control Theory and Applications 9 (September 2015) 2165-2170.

[13] Z. Ding and Z. Li, Distributed adaptive consensus control of nonlinear output-feedback systems on directed graphs, Automatica 72 (2016) $46-52$.

[14] Z. Li, W. Ren, X. Liu and M. Fu, Consensus of multiagent systems with general linear and lipschitz nonlinear dynamics using distributed adaptive protocols, IEEE Transactions on Automatic Control 58 (July 2013) 1786-1791.
[15] Z. Ding, Consensus disturbance rejection with disturbance observers, IEEE Transactions on Industrial Electronics 62 (Sept 2015) 5829-5837.

[16] H. Du, S. Li and C. Qian, Finite-time attitude tracking control of spacecraft with application to attitude synchronization, IEEE Transactions on Automatic Control 56 (Nov 2011) 2711-2717.

[17] F. Xiao, L. Wang, J. Chen and Y. Gao, Finite-time formation control for multi-agent systems, Automatica 45(11) (2009) 2605-2611.

[18] M. Nazari, E. Butcher, T. Yucelen and A. Sanyal, Decentralized consensus control of a rigid-body spacecraft formation with communication delay, Journal of Guidance, Control, and Dynamics 39(4) (2016) 838851.

[19] I. Ali, G. Radice and J. Kim, Backstepping control design with actuator torque bound for spacecraft attitude maneuver, Journal of Guidance, Control, and Dynamics 33(1) (2010) 254-259.

[20] H. Zhang and J. Fang, Robust backstepping control for agile satellite using double-gimbal variable-speed control moment gyroscope, Journal of Guidance, Control, and Dynamics 36 (September-October 2013) 13561363.

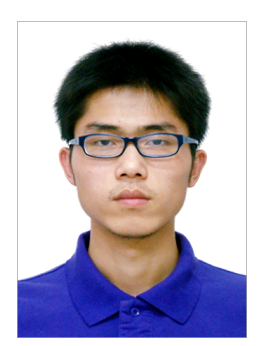

Yuanjin Yu received his B.S. degree in automation from Huaqiao University, China, and his Ph.D. degree in precision instruments and machinery from Beihang University, China, , in 2008 and 2015, respectively. From 2015 to 2017, he was postdoctoral fellow at Beihang University. From 2017 to 2018, he was at University of Manchester, as Research Associate. He is currently Lecturer in School of Mechanical Engineering, University of Science and Technology Beijing.

Yuanjin $\mathrm{Yu}$ is the author of over 15 technical publications and proceedings. His research interests include control theory and engineering applications, multi-agents formation.

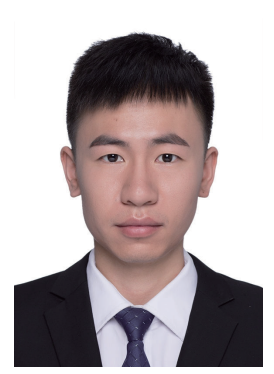

Zhenhong Li received his B.Eng. degree in electrical engineering from Huazhong University 
of Science and Technology, Hubei, China, in 2013, and the M.Sc. degree in control systems from the University of Manchester, Manchester, U.K., in 2014. He is now a $\mathrm{Ph} . \mathrm{D}$. candidate in control engineering with the School of Electrical and Electronic Engineering at the University of Manchester, U.K. His research interests include distributed optimization, and cooperative control of multi-agent systems.

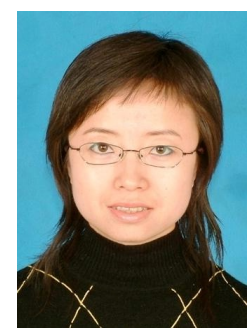

Zhaohua Yang received her M.S. degree control theory and engineering from Lanzhou University of Technology, China, and her Ph.D. degree in precision instruments and machinery from Harbin Institute of Technology, China, in 2001 and 2004, respectively. From 2004 to 2006, she was postdoctoral fellow at Beihang University. She is currently associate professor in School of Instrumentation Science and Opto-electronics Engineering, Beihang University.

Zhaohua Yang is the author of over 50 technical publications and proceedings. Her research interests include mathematic modeling and systems engineering, control theory and engineering application.

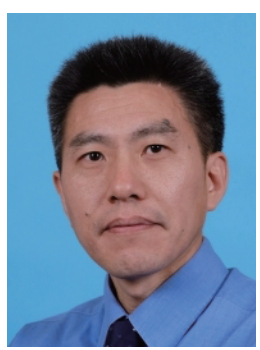

Zhengtao Ding received the B.Eng. degree from Tsinghua University, Beijing, China, and the M.Sc. degree in systems and control and the Ph.D. degree in control systems from the University of Manchester Institute of Science and Technology, Manchester, U.K.

After working as a Lecturer with Ngee Ann Polytechnic, Singapore, for ten years, in 2003, he joined The University of Manchester, Manchester, U.K., where he is currently Professor of Control Systems with the School of Electrical and Electronic Engineering. He is the author of the book Nonlinear and Adaptive Control Systems (IET, 2013) and a number of journal papers. His research interests include nonlinear and adaptive control theory and their applications.

Prof. Ding serves as an Associate Editor for the IEEE Control Systems Letters, Transactions of the Institute of Measurement and Control, Control Theory and Technology, Mathematical Problems in Engineering, Unmanned System$s$, and the International Journal of Automation and Computing. 